\title{
Serial Changes of Neointimal Tissue after Everolimus-Eluting Stent Implantation in Porcine Coronary Artery: An Optical Coherence Tomography Analysis
}

\author{
Hoyoun Won, ${ }^{1}$ Jung-Sun Kim, ${ }^{2,3}$ Dong-Ho Shin, ${ }^{2,3}$ Byeong-Keuk Kim, ${ }^{2,3}$ Young-Guk Ko, ${ }^{2,3}$ \\ Donghoon Choi, ${ }^{2,3}$ Yangsoo Jang, ${ }^{2,3,4}$ and Myeong-Ki Hong ${ }^{2,3,4}$ \\ ${ }^{1}$ Chung-Ang University Medical Center, 102 Heukseokro, Dongjak-gu, Seoul 156-755, Republic of Korea \\ ${ }^{2}$ Division of Cardiology, Department of Internal Medicine, Severance Cardiovascular Hospital, Yonsei University College of Medicine, \\ Yonsei University Health System, 250 Seongsanno, Seodaemun-go, Seoul 120-752, Republic of Korea \\ ${ }^{3}$ Cardiovascular Institute, Yonsei University College of Medicine, 250 Seongsanno, Seodaemun-go, Seoul 120-752, Republic of Korea \\ ${ }^{4}$ Severance Biomedical Science Institute, Yonsei University College of Medicine, 250 Seongsanno, Seodaemun-go, \\ Seoul 120-752, Republic of Korea
}

Correspondence should be addressed to Myeong-Ki Hong; mkhong61@yuhs.ac

Received 22 July 2014; Revised 27 August 2014; Accepted 4 September 2014; Published 18 September 2014

Academic Editor: Pablo Avanzas

Copyright () 2014 Hoyoun Won et al. This is an open access article distributed under the Creative Commons Attribution License, which permits unrestricted use, distribution, and reproduction in any medium, provided the original work is properly cited.

Purposes. The serial changes in neointimal tissues were compared between everolimus-eluting stent (EES) and bare-metal stent (BMS) in the porcine coronary artery using optical coherence tomography (OCT). Methods. Serial (1, 3, and 6 month followup after stent implantation) OCT examinations were performed in 15 swine with 15 BMS- and 15 EES-treated lesions in porcine coronary arteries. Results. In BMS-implanted lesions, neointimal volume decreased from $7.3 \mathrm{~mm}^{3}$ to $6.9 \mathrm{~mm}^{3}$ and $6.4 \mathrm{~mm}^{3}$ at 1 , 3 , and 6 months follow-up without statistical significance $(P=0.369)$. At the time points of 1,3 , and 6 months, neointimal tissue appearance was mainly a homogeneous pattern $(80.0 \%, 93.3 \%$, and $100 \%$, resp.), while the other pattern was layered. In contrast, in EES-implanted lesions, neointimal volume significantly increased from $4.8 \mathrm{~mm}^{3}$ to $9.8 \mathrm{~mm}^{3}$ between 1 and 3 months but significantly decreased to $8.6 \mathrm{~mm}^{3}$ between 3 and 6 months $(P<0.001)$. Between 1 and 3 months, the layered pattern of neointimal tissue increased from $26.7 \%$ to $66.7 \%$ but decreased to $20.0 \%$ between 3 and 6 months. Conclusions. EES had a biphasic pattern of neointimal amounts that correlated with changes in neointimal morphology.

\section{Introduction}

The development of drug-eluting stents (DES) has significantly reduced the incidence of in-stent restenosis compared to bare-metal stents (BMS) $[1,2]$. However, even in the DES era, in-stent restenosis remains a significant clinical problem, particularly in high-risk patients [3]. Optical coherence tomography (OCT) is a high-resolution imaging tool that is ideal for the evaluation of neointimal tissue characteristics as well as strut coverage of coronary stents [4-6]. The everolimus-eluting stent (EES) is a second-generation DES with a target lesion revascularization rate as low as $4.7 \%$ during the 5-year clinical follow-up [7]. However, there are few studies evaluating temporal changes in neointimal tissue after EES implantation. The purpose of this study was to evaluate serial changes in neointimal tissue in stented segments based on OCT between 1, 3, and 6 months after EES implantation compared to BMS implantation in normal porcine coronary arteries.

\section{Materials and Methods}

2.1. Study Design. A total of 15 swine (weighing 25 to $30 \mathrm{~kg}$ ) were studied for 6 months to evaluate neointimal tissue serially following EES implantation. Two stent types were implanted: EES (Xience Prime, $3.0 \times 12 \mathrm{~mm}$, Abbott Vascular, Santa Clara, CA) as a target stent and BMS 


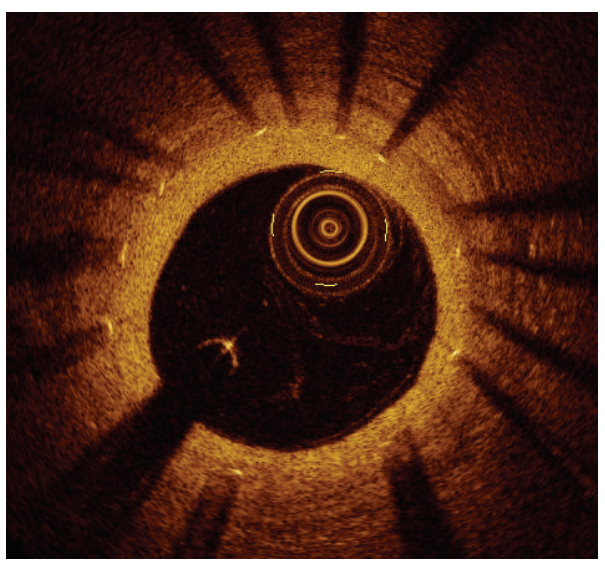

(a)

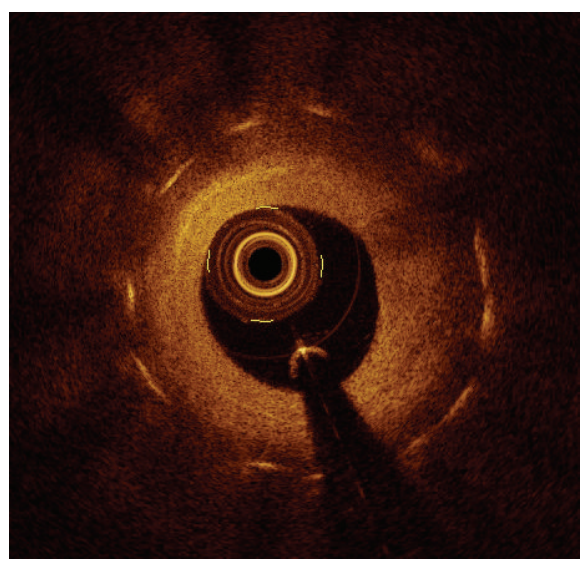

(b)

FIGURE 1: Representative optical coherence tomographic images of neointima. Homogeneous pattern (a) and layered pattern (b).

(Blazer, $3.0 \times 13 \mathrm{~mm}$, OrbusNeich, Hong Kong) as a control stent. Each stent was deployed in the left anterior descending artery or right coronary artery. At the time of stent deployment, each stent was systematically randomized to each coronary artery in each animal. Follow-up angiography with OCT was serially performed 1,3 , and 6 months after stent implantation. All animals received humane care in compliance with the Animal Welfare Act and "The Guide for the Care and Use of Laboratory Animals" formulated by the Institute of Laboratory Animal Research [8]. This study was approved by the local institutional animal care and use committee (Medi Kinetics, MK-IACUC: 111027-0001 and Cardiovascular Production Evaluation Center, Yonsei University College of Medicine).

2.2. Procedural Description. All animals were premedicated at least 12 hours before the procedure with $100 \mathrm{mg}$ aspirin and $300 \mathrm{mg}$ clopidogrel. Anesthesia was performed via intramuscular injection of ketamine $(20 \mathrm{mg} / \mathrm{kg})$ and xylazine $(2 \mathrm{mg} / \mathrm{kg})$. After adequate anesthesia, animals were intubated and inhaled isoflurane (1-2\%) delivered through a precision vaporizer and a circle absorption breathing system with periodic arterial blood gas monitoring. Under sterile conditions, arteriotomy of the carotid artery was performed and a 6-Fr vascular access sheath was introduced into the carotid artery. Vital signs were continuously monitored using surface electrocardiography and were recorded at approximately $20 \mathrm{~min}$ intervals. Unfractionated heparin (5,000 to $10,000 \mathrm{IU})$ was administered to maintain activated clotting times of 250 to 300 seconds. Using fluoroscopic guidance, stent implantation on coronary arteries was performed with a stent-to-artery ratio of $110 \%$ to $120 \%$ overstretch for full apposition at predetermined sites using conventional techniques. After stent implantation, carotid arteries were repaired and the incision site was closed with adequate suture material until the next use of the carotid arteries. All animals received $100 \mathrm{mg}$ aspirin and $75 \mathrm{mg}$ clopidogrel daily after stent implantation. They were fed a regular diet throughout the duration of the study [4].
2.3. OCT Procedure and Analyses. OCT was performed using the C7-XR imaging systems (LightLab Imaging, Inc., St. Jude Medical, St. Paul, MN). The OCT catheter was pulled back at $20 \mathrm{~mm} / \mathrm{s}$ and OCT images were generated at $100 \mathrm{frames} / \mathrm{s}$. Contrast media were continuously flushed through a guiding catheter at a rate of 4 to $5 \mathrm{~mL} / \mathrm{s}$ for 3 to 4 seconds. Images were continuously acquired and stored digitally for subsequent analyses. All OCT images were analyzed at a core laboratory (Cardiovascular Research Center, Seoul, Republic of Korea) by analysts who were blinded to procedural information. Cross-sectional OCT images were analyzed at $1 \mathrm{~mm}$ intervals. Stent and luminal cross-sectional areas (CSA) were measured and neointimal hyperplasia (NIH) CSA was calculated by subtracting the luminal CSA from the stent CSA. The NIH CSA percentage was calculated as the NIH CSA divided by the stent CSA. NIH thickness was measured as the distance between the strut with a line perpendicular to the neointima and the endoluminal surface of the neointima. The NIH volume was calculated as NIH CSA $\times$ length. The maximal NIH site with $2 \mathrm{~mm}$ proximal and distal adjacent portion in the stented segment was serially compared to the corresponding segment based on the maximal NIH site at the 1 month follow-up. Serial comparisons were performed by matching the length from the stent edge and anatomical features, such as side branch.

If the mean neointimal thickness was $\geq 20 \mu \mathrm{m}$, neointimal tissue characteristics were qualitatively classified as homogeneous, heterogeneous, or layered pattern. Homogeneous patterns were defined as uniform optical neointima tissue properties without focal variation in backscattering pattern. Heterogeneous patterns were defined as focal changes in optical properties and various backscattering patterns. Layered pattern refers to concentric layers with different optical properties, namely, an adluminal high scattering layer and abluminal low scattering layer (Figure 1) [9].

2.4. Quantitative Angiographic Analyses. Quantitative coronary angiography analyses were performed using an offline computerized quantitative coronary angiographic system 

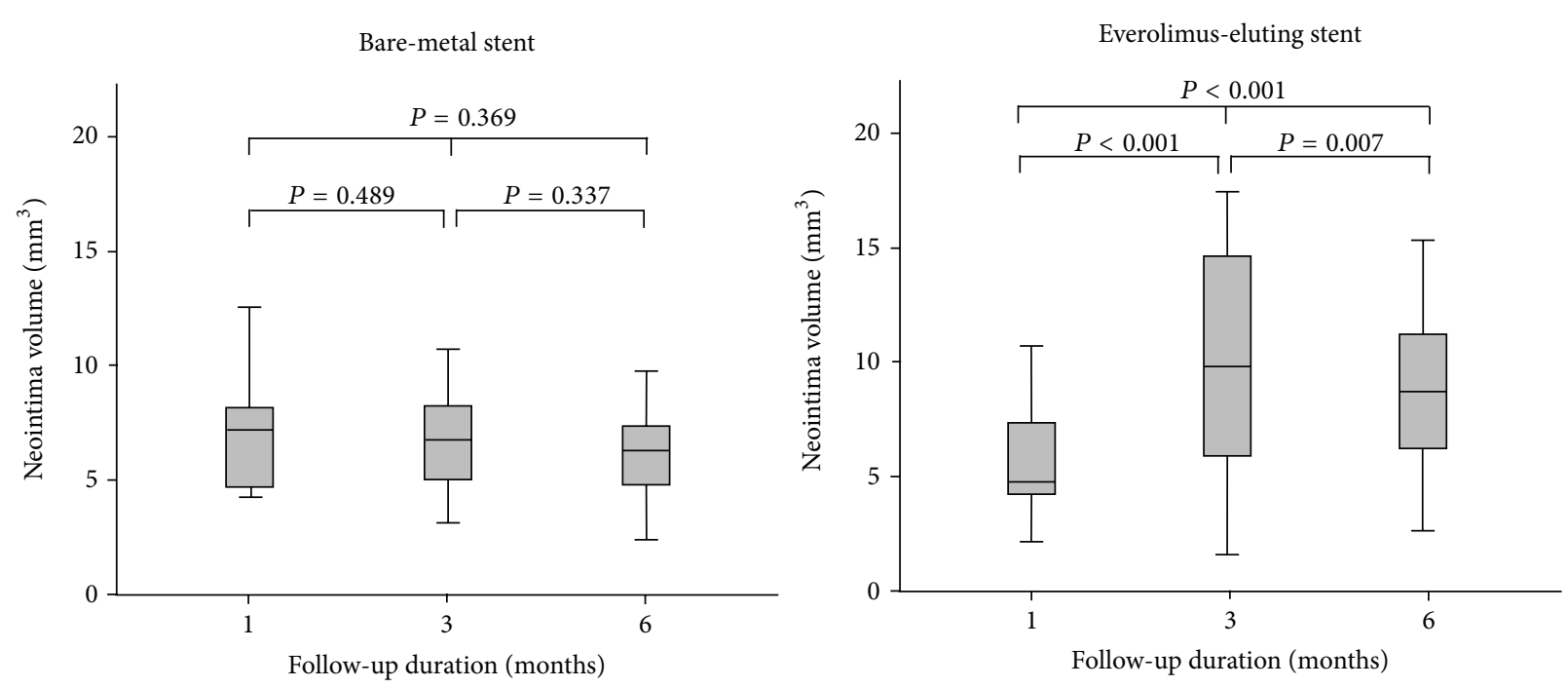

FIGURE 2: Serial changes in neointimal volume at the segment with maximal amounts of neointimal hyperplasia at the 1-month follow-up.

(CASS System, Pie Medical Imaging, Maastricht, The Netherlands) in an independent core laboratory (Cardiovascular Research Center, Seoul, Korea). The minimal lumen diameter and reference diameters of treated coronary lesions were measured in the view with the narrowest lumen and the least amount of foreshortening.

2.5. Statistical Analyses. Continuous variables are expressed as a median with interquartile range (IQR). Wilcoxon signedrank tests or Friedman tests were used to compare variables in a longitudinal manner. Categorical variables are expressed as both numbers and percentages and were compared using Fisher's exact tests. Data were analyzed using SPSS 18.0 software for Windows (SPSS, Chicago, IL). A $P$ value $<0.05$ was considered statistically significant.

\section{Results and Discussion}

3.1. Results. EES was implanted in eight left anterior descending arteries and seven right coronary arteries, whereas BMS was deployed in seven left anterior descending arteries and eight right coronary arteries. Balloon-to-artery ratio was similar between two groups (1.11 \pm 0.06 in BMS versus $1.14 \pm$ 0.04 in EES, $P=0.189$ ). Serial OCT evaluation of $\mathrm{NIH}$ was assessed in a total of 15 BMS- and 15 EES-implanted into the coronary arteries of 15 animals after 1, 3, and 6 months. Serial angiographic and OCT findings are shown in Table 1. Based on analyses of whole stented segments in BMS, the mean NIH thickness was maximal at 1 month (190.4 $\mu \mathrm{m}, \mathrm{IQR} 127.0-243.9 \mu \mathrm{m})$ and decreased at 3 and 6 months $(182.2 \mu \mathrm{m}$, IQR $131.9-229.4 \mu \mathrm{m}$ and $169.8 \mu \mathrm{m}$, IQR $112.7-211.1 \mu \mathrm{m}, P=0.189)$. However, in EES, it was maximal at 3 months $(276.5 \mu \mathrm{m}$, IQR $172.2-428.1 \mu \mathrm{m})$ and decreased at 6 months $(269.6 \mu \mathrm{m}$, IQR 199.9-396.4 $\mu \mathrm{m}, P=0.100)$. For analyses of maximal NIH segments at 1 month, serial changes in NIH volume after BMS and EES are shown in Figure 2 and Table 2. In BMS-implanted lesions, NIH volume decreased

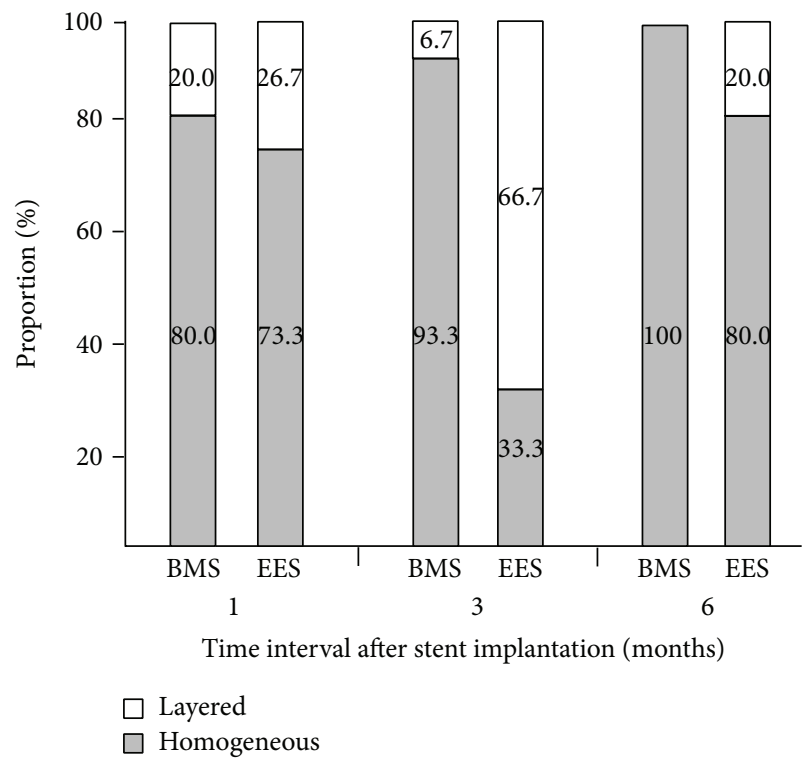

FIGURE 3: Serial changes in neointimal patterns at the segment with maximal amounts of neointimal hyperplasia at the 1-month followup. BMS, bare-metal stent; EES, everolimus-eluting stent.

from $7.3 \mathrm{~mm}^{3}$ (IQR $4.7-8.4 \mathrm{~mm}^{3}$ ) at the 1-month follow-up to $6.9 \mathrm{~mm}^{3}$ (IQR 4.6-8.2 $\mathrm{mm}^{3}$ ) and $6.4 \mathrm{~mm}^{3}$ (IQR 4.3-7.4 $\mathrm{mm}^{3}$ ) after 3 and 6 months, respectively $(P=0.369)$. At the different time points of 1,3 , and 6 months, neointimal tissue appearance was predominantly the homogeneous pattern $(80.0 \%, 93.3 \%$, and $100 \%$, resp.), while the other pattern was layered (Figure 3). In contrast, in EES-implanted lesions, NIH volume significantly increased from $4.8 \mathrm{~mm}^{3}$ (IQR 4.1$7.6 \mathrm{~mm}^{3}$ ) to $9.8 \mathrm{~mm}^{3}$ (IQR $5.7-15.1 \mathrm{~mm}^{3}$ ) between 1 and 3 months $(P<0.001)$ but significantly decreased to $8.6 \mathrm{~mm}^{3}$ (IQR $\left.6.0-11.2 \mathrm{~mm}^{3}\right)$ between 3 and 6 months $(P=0.007)$ $(P<0.001$ between 1 and 6 months) (Figure 2). Between 


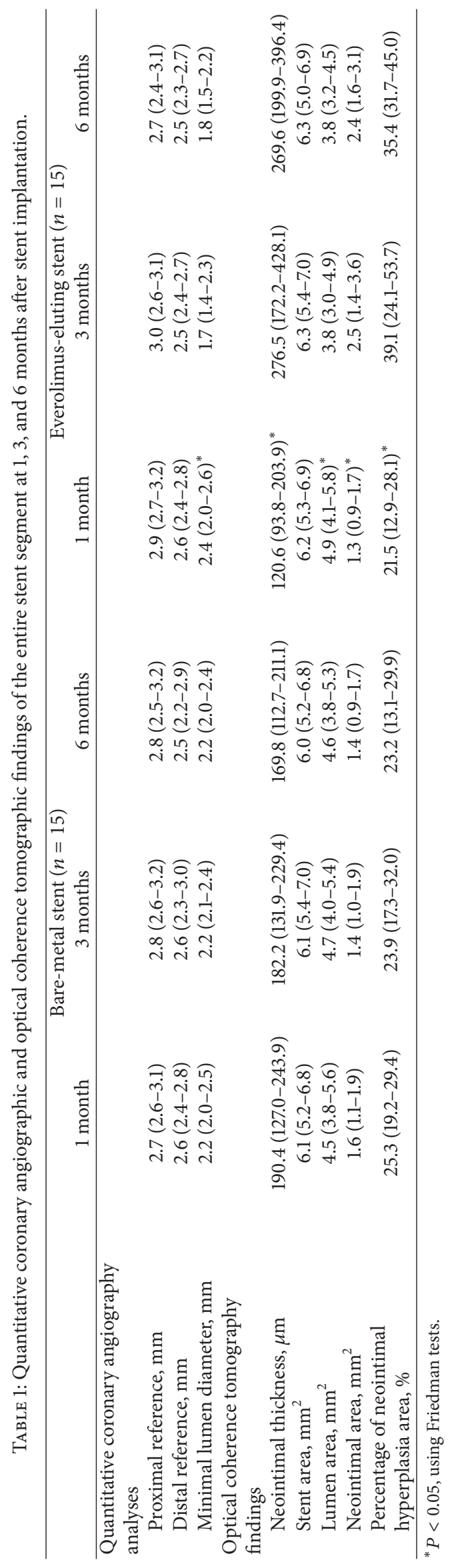


TABLE 2: Optical coherence tomographic findings of the maximum neointima site at 1,3, and 6 months after stent implantation.

\begin{tabular}{|c|c|c|c|c|c|c|}
\hline & \multicolumn{3}{|c|}{ Bare-metal stent $(n=15)$} & \multicolumn{3}{|c|}{ Everolimus-eluting stent $(n=15)$} \\
\hline & 1 month & 3 months & 6 months & 1 month & 3 months & 6 months \\
\hline \multicolumn{7}{|l|}{ Quantitative analyses } \\
\hline Stent volume, $\mathrm{mm}^{3}$ & $\begin{array}{c}24.5 \\
(21.4-27.4)\end{array}$ & $\begin{array}{c}24.3 \\
(21.8-26.9)\end{array}$ & $\begin{array}{c}23.3 \\
(21.4-26.5)\end{array}$ & $\begin{array}{c}25.5 \\
(20.7-27.2)\end{array}$ & $\begin{array}{c}25.5 \\
(20.4-27.6)\end{array}$ & $\begin{array}{c}24.7 \\
(19.9-27.9)\end{array}$ \\
\hline Lumen volume, $\mathrm{mm}^{3}$ & $\begin{array}{c}16.7 \\
(14.1-20.2)\end{array}$ & $\begin{array}{c}17.5 \\
(15.7-20.6)\end{array}$ & $\begin{array}{c}17.6 \\
(14.4-20.8)\end{array}$ & $\begin{array}{c}18.7 \\
(16.5-21.4)\end{array}$ & $\begin{array}{c}12.6 \\
(11.5-17.6)\end{array}$ & $\begin{array}{c}15.5 \\
(13.3-18.3)\end{array}$ \\
\hline Neointima volume, $\mathrm{mm}^{3}$ & $7.3(4.7-8.4)$ & $6.9(4.6-8.2)$ & $6.4(4.3-7.4)$ & $4.8(4.1-7.6)$ & $9.8(5.7-15.1)$ & $8.6(6.0-11.2)$ \\
\hline \multicolumn{7}{|l|}{ Qualitative analyses } \\
\hline Homogeneous, \% & $12(80.0)$ & $14(93.3)^{*}$ & $15(100)$ & $11(73.3)$ & $5(33.3)$ & $12(80.0)$ \\
\hline Layered, \% & $3(20.0)$ & $1(6.7)^{*}$ & $0(0)$ & $4(26.7)$ & $10(66.7)$ & $3(20.0)$ \\
\hline
\end{tabular}

${ }^{*} P<0.05$, comparing bare-metal stent with everolimus-eluting stent.
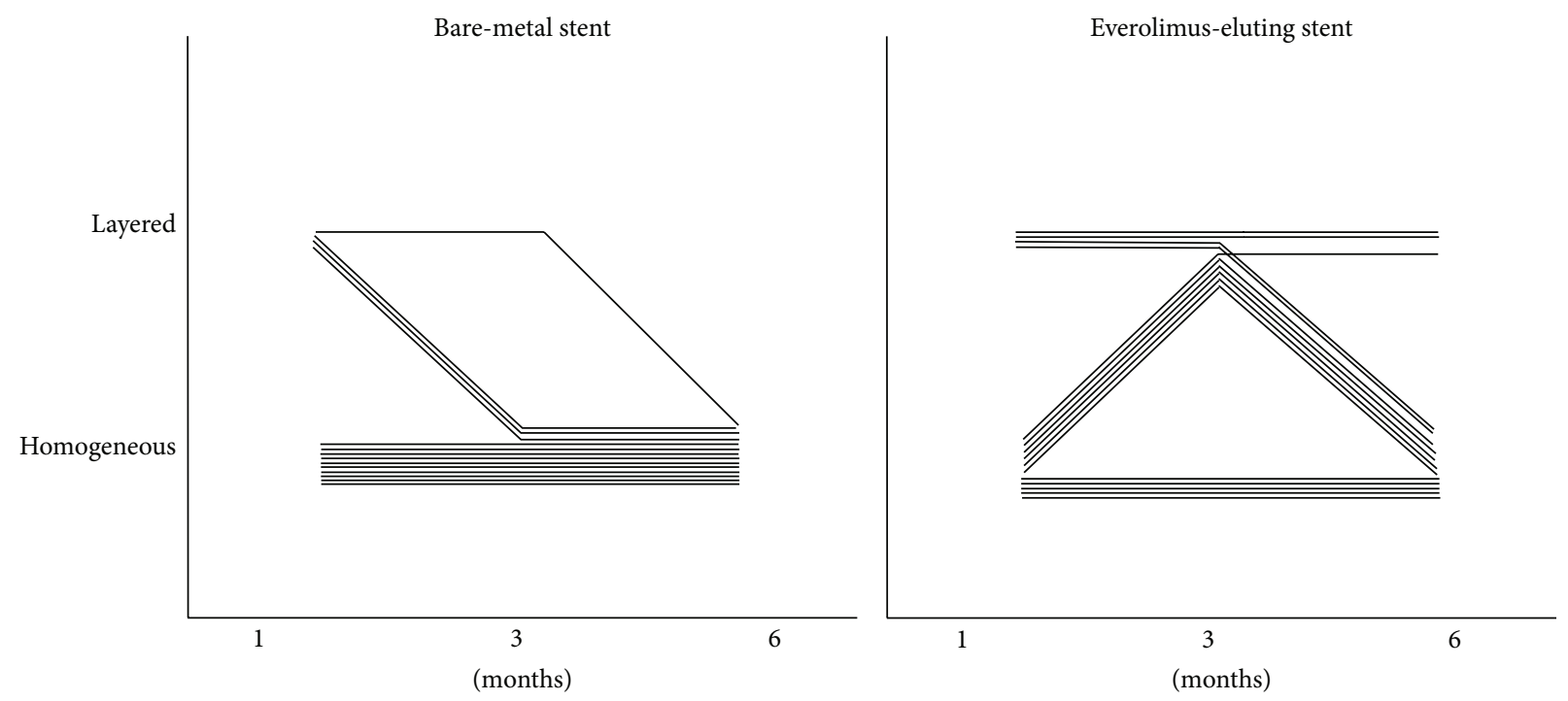

FIGURE 4: Evolution of serial changes in neointimal patterns in bare-metal stent and everolimus-eluting stent.

1 and 3 months, the layered pattern of neointima increased from $26.7 \%$ to $66.7 \%$ but decreased to $20.0 \%$ between 3 and 6 months (Figures 3 and 4). Figures 5 and 6 show typical OCT images of serial NIH changes in BMS- and EES-implanted coronary arteries, respectively.

3.2. Discussion. Using serial (1, 3, and 6 months followup) OCT in the nonatherosclerotic porcine coronary artery model, the present study showed that (1) a biphasic neointimal response of EES consisted of an early progression phase and late regression phase and (2) quantitative neointima changes correlated with morphological neointima changes from a layered pattern to a homogeneous pattern.

Serial quantitative neointimal changes of BMS have been well evaluated in human studies. In BMS, long-term angiographic luminal responses were a triphasic pattern that consisted of a restenosis phase at the early period for 6 months, a regression phase at the intermediate period between 6 months and 3 years, and a late renarrowing phase after 4 years [10]. The results of intravascular ultrasound studies were also consistent with those from a previous angiographic study [11, 12]. One intravascular ultrasound study reported that mean neointimal CSA significantly decreased from $2.6 \pm 1.0$ to $2.3 \pm$ $0.9 \mathrm{~mm}^{2}$ between 6 and 24 months after BMS-implantation [12]. The results from the BMS-implanted lesions in the current study are in agreement with results from previous studies [10-12].

However, information regarding long-term serial changes in neointima following DES implantation was not sufficient. Sousa et al. first reported after 4-year angiographic follow-up of 26 sirolimus-eluting stent-treated patients that a temporal progression of late loss was observed: late loss was $0.08 \mathrm{~mm}$ from postintervention to the 1-year follow-up, and it was $-0.03 \mathrm{~mm}$ from 1- to 2-years and $0.20 \mathrm{~mm}$ from 2- to 4 -years [13]. In another study with 15 sirolimus-eluting stent-treated patients, there were no significant changes in stent minimal lumen diameter based on quantitative coronary angiographic analyses and no significant deterioration in lumen volume by intravascular ultrasound measurement between the 6-month and 2-year follow-up [14]. In substudy of randomized TAXUS 


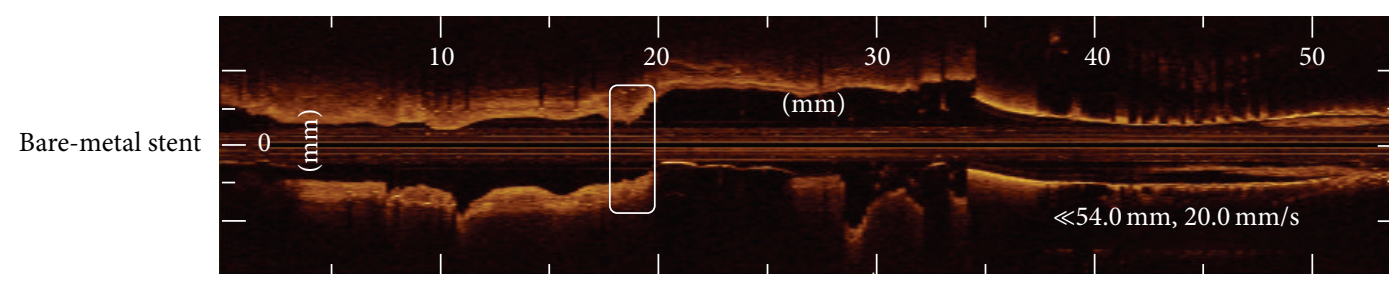

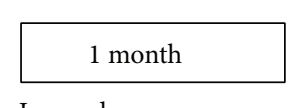

Layered

NIH volume. $10.0 \mathrm{~mm}^{3}$

\begin{tabular}{l}
\hline 3 months \\
Homogeneous \\
NIH volume. $8.8 \mathrm{~mm}^{3}$
\end{tabular}

6 months

Homogeneous

NIH volume. $6.4 \mathrm{~mm}^{3}$
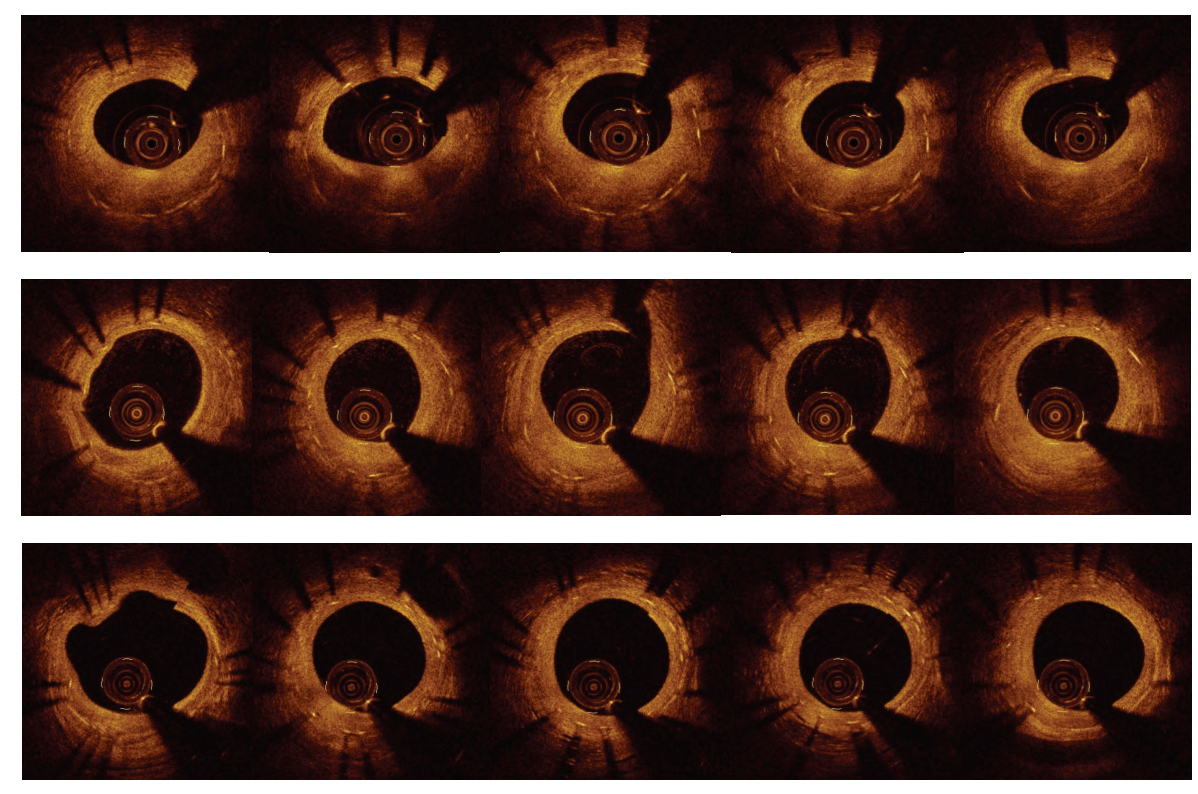

FIGURE 5: Examples of neointimal volume and patterns at the segment with maximal amounts of neointimal hyperplasia at 1, 3, and 6 months following bare-metal stent implantation. NIH, neointimal hyperplasia.

II trial, neointimal volume measured by intravascular ultrasound increased significantly from $10.13 \mathrm{~mm}^{3}$ at 6 months to $15.11 \mathrm{~mm}^{3}$ at 2 years after paclitaxel-eluting stent implantation $(P=0.0011)$ [15]. In 2-year follow-up results of the SPIRIT II trial, in-stent neointimal volume progressed from $4.13 \mathrm{~mm}^{3}$ at 6 months to $8.42 \mathrm{~mm}^{3}$ at 2 years after EES implantation [16].

Quantitative OCT assessment has been well validated. One study of in vivo OCT comparison with histology in a rabbit carotid model showed that histological and OCT measurements of mean $\mathrm{NIH}$ thickness were similar and closely related $(r=0.85, P<0.001)$ [17]. OCT and histology correlated highly for the evaluation of neointimal area $\left(R^{2}=\right.$ $0.804)$, luminal area $\left(R^{2}=0.825\right)$, and neointimal thickness $\left(R^{2}=0.789\right)$ in a normal porcine coronary model [4]. In qualitative OCT assessments, neointima with rich smooth muscle cells and dense collagen fibers has a high backscatter signal and is shown as high optical intensity, extracellular matrix or myxomatous tissue, such as proteoglycan or fibrin rich neointima with few smooth muscle cells, and has a low backscatter signal and is shown as a lucent signal [1820]. Although a homogeneous pattern with a high optical intensity is usually observed in in-stent restenosis of BMS, the layered pattern was observed more frequently in in-stent restenosis of DES [18]. The layered pattern consisted of a high intense inner layer with smooth muscle cells and a low intense outer layer with extracellular material rich lesions [18]. In current study, heterogeneous pattern was not detected in contrast to the previous study [20], which could not be explained exactly. The previous study used hypercholesterolemic swine model and different type of DES (especially, paclitaxel-eluting stent), which was evident difference compared to our study [20].

Using intravascular imaging tools, serial follow-up data beyond 2 years after DES implantation are sparse in clinical practice as well as in random clinical trials due to the practical or ethical aspects of clinical practice. However, these data are very important for understanding the long-term natural history of DES-treated patients. Considering the findings that the neointimal growth pattern in porcine coronary stents after 1 month was compatible with those in human coronary stents after 6-12 months [21], the results of the present serial study (1, 3, and 6 months follow-up after stent implantation) in porcine coronary arteries may be compatible with very late (beyond approximately 2 or 3 years follow-up) neointimal responses in DES-treated patients. In the present study, NIH thickness in the analyses of whole segments and NIH volume in the analyses of maximal NIH segments in EES-implanted lesions were maximal at 3 months and decreased at 6 months. 

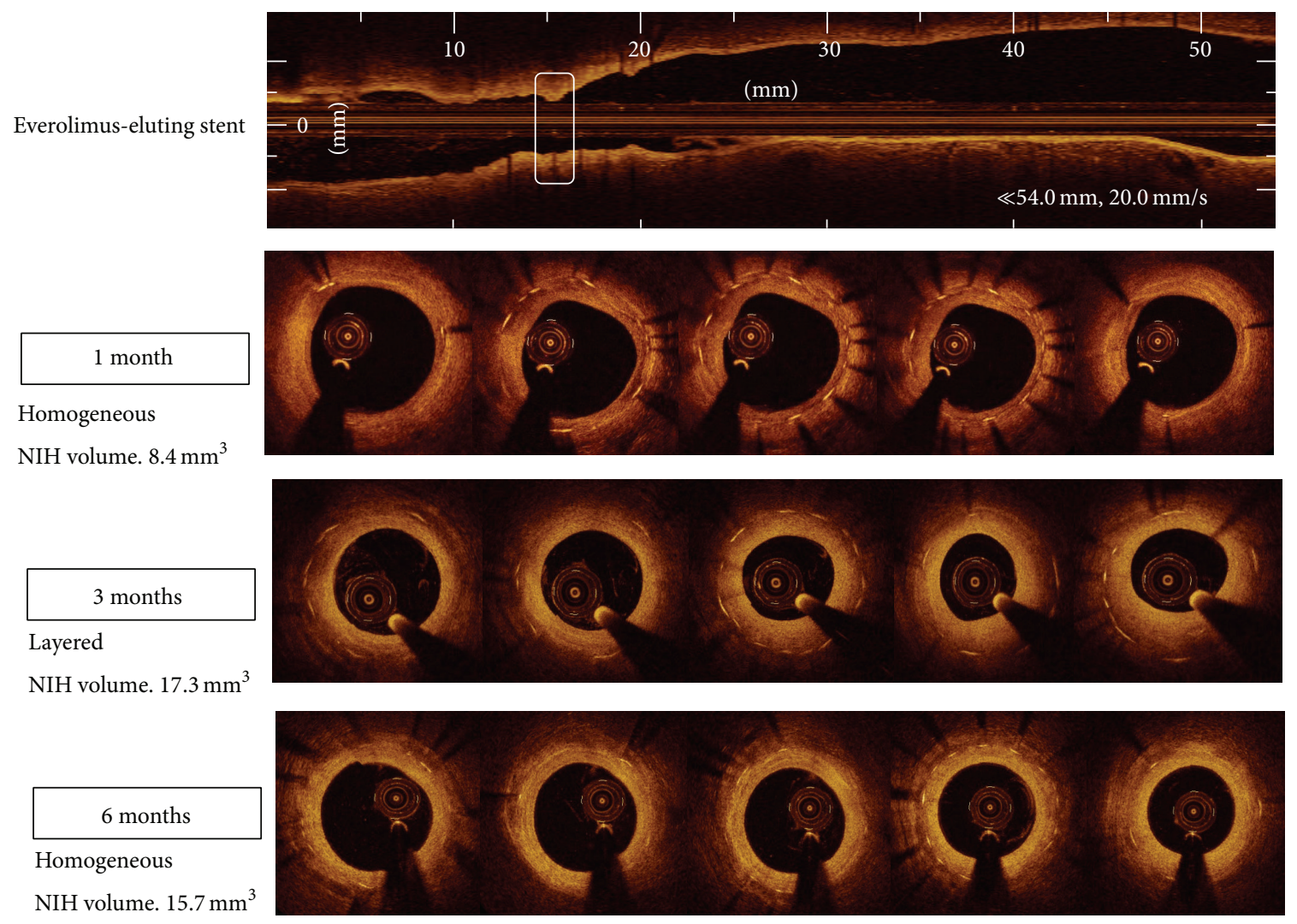

FIGURE 6: Examples of neointimal volume and patterns at the segment with maximal amounts of neointimal hyperplasia at 1,3, and 6 months following everolimus-eluting stent implantation. NIH, neointimal hyperplasia.

To the best of our knowledge, this is the first report to show a biphasic neointimal response of EES-implanted segments, particularly neointimal regression between 3 and 6 months in the porcine coronary model. In addition, neointimal regression occurred with a morphological shift from a layered pattern to a homogeneous pattern. The mechanism of the neointimal regression is not clearly understood. Recent OCT study reported that the evolution of heterogeneous to homogeneous neointima was observed in lesions with $\mathrm{NIH}$ regression, while the evolution of homogeneous to layered neointima was detected in lesions with $\mathrm{NIH}$ progression $(P<0.001)[22]$. The present study supports the notion that neointimal stabilization from immature tissue, such as fibrin near strut, rich extracellular matrix, and inflammatory cells to maturation with rich smooth muscle cells, may play a role in neointimal regression [23].

Limitations. This study is based on the nonatherosclerotic normolipemic porcine coronary model. Therefore, the results of the present study may need to be interpreted with caution for clinical practice of DES-treated patients. Although the diseased porcine animal models have been increasingly used for the evaluation of DES and provided more similar biological responses, normolipemic swine coronary artery is still recommended as the choice of the porcine model to evaluate tissue response in consensus documents [24, 25].
The comparisons between OCT images with histology were not performed at each stage of follow-up.

\section{Conclusion}

This serial OCT study found that EES had a biphasic pattern (early progression and late regression) of neointimal tissue that correlated with changes in neointimal morphology.

\section{Conflict of Interests}

The authors declare that there is no conflict of interests regarding the publication of this paper.

\section{Authors' Contribution}

Hoyoun Won and Jung-Sun Kim contributed equally to this study.

\section{Acknowledgments}

This study was supported by a Grant from the Korea Healthcare Technology R\&D Project, Ministry for Health, Welfare and Family Affairs, Republic of Korea (nos. A085012 and A102064), and Grants from the Korea Health 21 R\&D Project, 
Ministry of Health and Welfare, Republic of Korea (no. A085136) and the Cardiovascular Research Center, Seoul, Republic of Korea.

\section{References}

[1] A. J. Kirtane, A. Gupta, S. Iyengar et al., "Safety and efficacy of drug-eluting and bare metal stents: comprehensive metaanalysis of randomized trials and observational studies," Circulation, vol. 119, no. 25, pp. 3198-3206, 2009.

[2] O. C. Marroquin, F. Selzer, S. R. Mulukutla et al., "A comparison of bare-metal and drug-eluting stents for off-label indications," The New England Journal of Medicine, vol. 358, no. 4, pp. 342352, 2008.

[3] F. Liistro, M. Fineschi, P. Angioli et al., "Effectiveness and safety of sirolimus stent implantation for coronary in-stent restenosis. The true (tuscany registry of sirolimus for unselected in-stent restenosis) registry," Journal of the American College of Cardiology, vol. 48, no. 2, pp. 270-275, 2006.

[4] A. Murata, D. Wallace-Bradley, A. Tellez et al., "Accuracy of optical coherence tomography in the evaluation of neointimal coverage after stent implantation," JACC: Cardiovascular Imaging, vol. 3, no. 1, pp. 76-84, 2010.

[5] F. Prati, E. Regar, G. S. Mintz et al., "Expert review document on methodology, terminology, and clinical applications of optical coherence tomography: Physical principles, methodology of image acquisition, and clinical application for assessment of coronary arteries and atherosclerosis," European Heart Journal, vol. 31, no. 4, pp. 401-415, 2010.

[6] S.-Y. Lee and M.-K. Hong, "Stent evaluation with optical coherence tomography," Yonsei Medical Journal, vol. 54, no. 5, pp. 1075-1083, 2013.

[7] Y. Onuma, K. Miquel-Hebert, and P. W. Serruys, "Five-year long-term clinical follow-up of the XIENCE V everolimuseluting coronary stent system in the treatment of patients with de novo coronary artery disease: the SPIRIT II trial," EuroIntervention, vol. 8, no. 9, pp. 1047-1051, 2013.

[8] Institute of Laboratory Animal Resources (U.S.), Guide for the Care and Use of Laboratory Animals, National Academy Press, Washington, DC, USA, 1996.

[9] N. Gonzalo, P. W. Serruys, T. Okamura et al., "Optical coherence tomography patterns of stent restenosis," The American Heart Journal, vol. 158, no. 2, pp. 284-293, 2009.

[10] T. Kimura, K. Abe, S. Shizuta et al., "Long-term clinical and angiographic follow-up after coronary stent placement in native coronary arteries," Circulation, vol. 105, no. 25, pp. 2986-2991, 2002.

[11] N. Kuroda, Y. Kobayashi, M. Nameki et al., "Intimal hyperplasia regression from 6 to 12 months after stenting," American Journal of Cardiology, vol. 89, no. 7, pp. 869-872, 2002.

[12] M.-K. Hong, C. W. Lee, Y.-H. Kim et al., "Two-year followup intravascular ultrasound analysis after bare metal stent implantation in 120 lesions," Catheterization and Cardiovascular Interventions, vol. 65, no. 2, pp. 247-253, 2005.

[13] J. E. Sousa, M. A. Costa, A. Abizaid et al., "Four-year angiographic and intravascular ultrasound follow-up of patients treated with sirolimus-eluting stents," Circulation, vol. 111, no. 18, pp. 2326-2329, 2005.

[14] M. Degertekin, P. W. Serruys, D. P. Foley et al., "Persistent inhibition of neointimal hyperplasia after sirolimus-eluting stent implantation: long-term (up to 2 years) clinical, angiographic, and intravascular ultrasound follow-up," Circulation, vol. 106, no. 13, pp. 1610-1613, 2002.

[15] K. Tsuchida, P. W. Serruys, N. Bruining et al., "Two-year serial coronary angiographic and intravascular ultrasound analysis of in-stent angiographic late lumen loss and ultrasonic neointimal volume from the TAXUS II trial," The American Journal of Cardiology, vol. 99, no. 5, pp. 607-615, 2007.

[16] B. E. Claessen, M. A. Beijk, V. Legrand et al., "Two-year clinical, angiographic, and intravascular ultrasound follow-up of the XIENCE V everolimus-eluting stent in the treatment of patients with de novo native coronary artery lesions the SPIRIT II trial," Circulation: Cardiovascular Interventions, vol. 2, no. 4, pp. 339347, 2009.

[17] F. Prati, M. Zimarino, E. Stabile et al., "Does optical coherence tomography identify arterial healing after stenting? An in vivo comparison with histology, in a rabbit carotid model," Heart, vol. 94, no. 2, pp. 217-221, 2008.

[18] H. Nagai, H. Ishibashi-Ueda, and K. Fujii, "Histology of highly echolucent regions in optical coherence tomography images from two patients with sirolimus-eluting stent restenosis," Catheterization and Cardiovascular Interventions, vol. 75, no. 6, pp. 961-963, 2010.

[19] H. Otake, J. Shite, F. Ikeno et al., "Evaluation of the peri-strut low intensity area following sirolimus- and paclitaxel-eluting stents implantation: insights from an optical coherence tomography study in humans," International Journal of Cardiology, vol. 157, no. 1, pp. 38-42, 2012.

[20] J.-S. Kim, M. E. Afari, J. Ha et al., "Neointimal patterns obtained by optical coherence tomography correlate with specific histological components and neointimal proliferation in a swine model of restenosis," European Heart Journal Cardiovascular Imaging, vol. 15, no. 3, pp. 292-298, 2014.

[21] R. Virmani, F. D. Kolodgie, A. Farb, and A. Lafont, "Drug eluting stents: are human and animal studies comparable?" Heart, vol. 89, no. 2, pp. 133-138, 2003.

[22] S. Y. Lee, M. K. Hong, G. S. Mintz et al., "Temporal course of neointimal hyperplasia following drug-eluting stent implantation: a serial follow-up optical coherence tomography analysis," The International Journal of Cardiovascular Imaging, vol. 30, pp. 1003-1011, 2014.

[23] T. Kimura, H. Yokoi, Y. Nakagawa et al., "Three-year followup after implantation of metallic coronary-artery stents," New England Journal of Medicine, vol. 334, no. 9, pp. 561-566, 1996.

[24] R. S. Schwartz, E. R. Edelman, A. Carter et al., "Drug-eluting stents in preclinical studies: recommended evaluation from a consensus group," Circulation, vol. 106, no. 14, pp. 1867-1873, 2002.

[25] R. S. Schwartz, E. Edelman, R. Virmani et al., "Drug-eluting stents in preclinical studies: updated consensus recommendations for preclinical evaluation," Circulation. Cardiovascular Interventions, vol. 1, no. 2, pp. 143-153, 2008. 


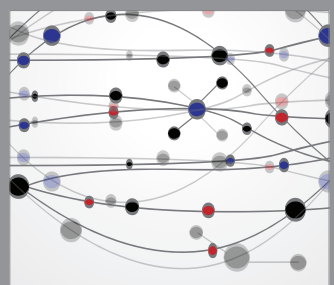

The Scientific World Journal
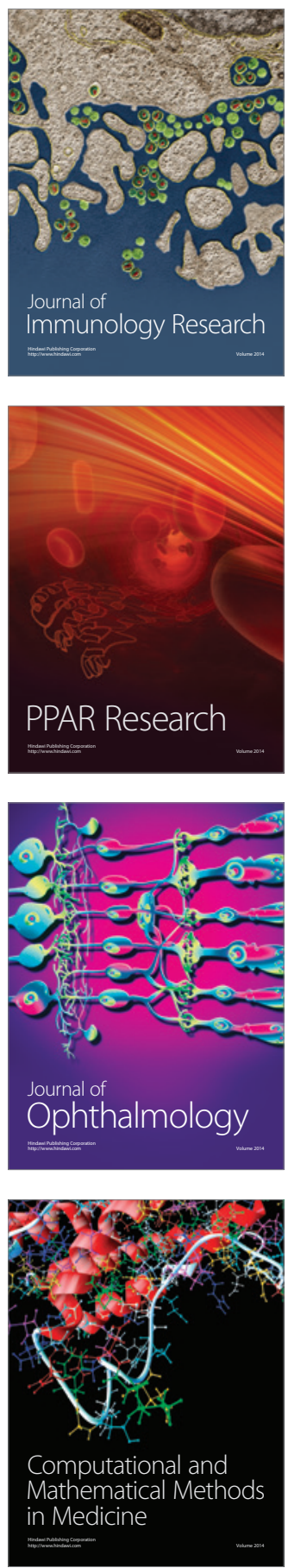

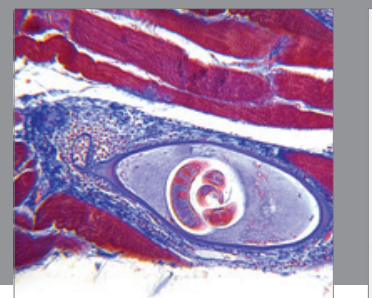

Gastroenterology

Research and Practice
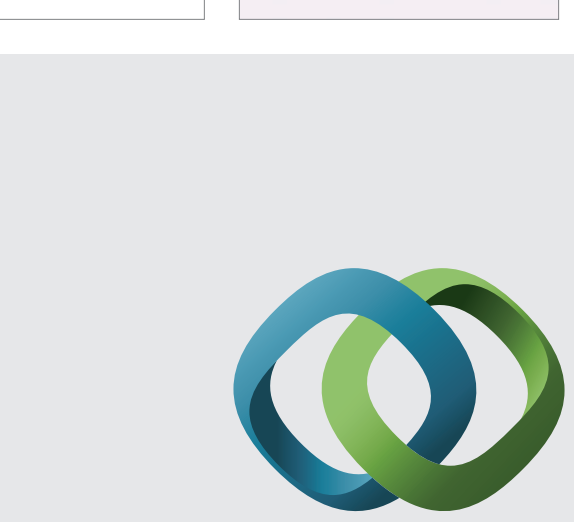

\section{Hindawi}

Submit your manuscripts at

http://www.hindawi.com
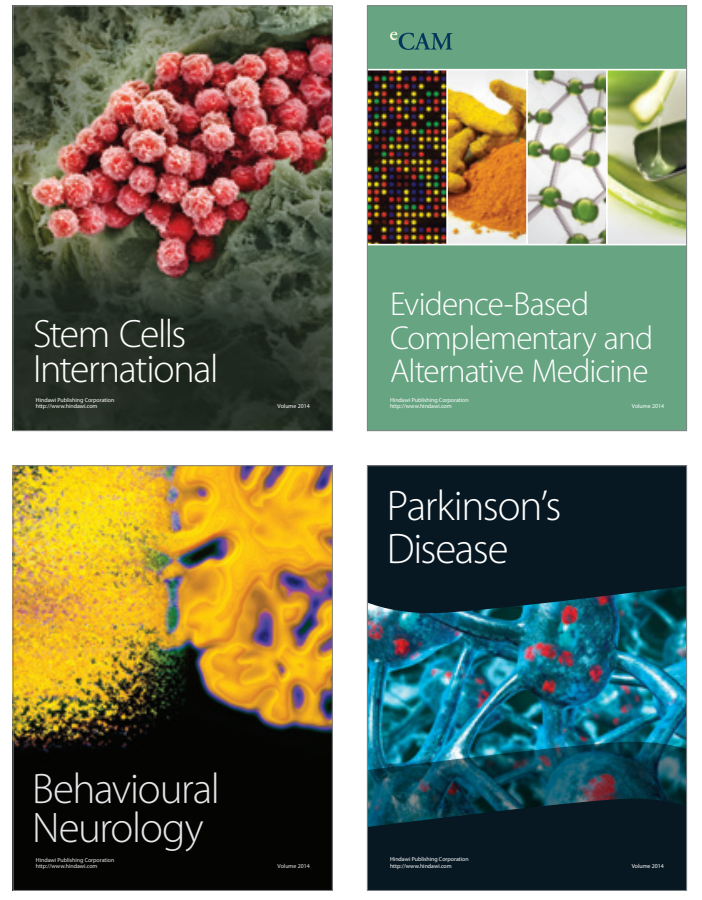
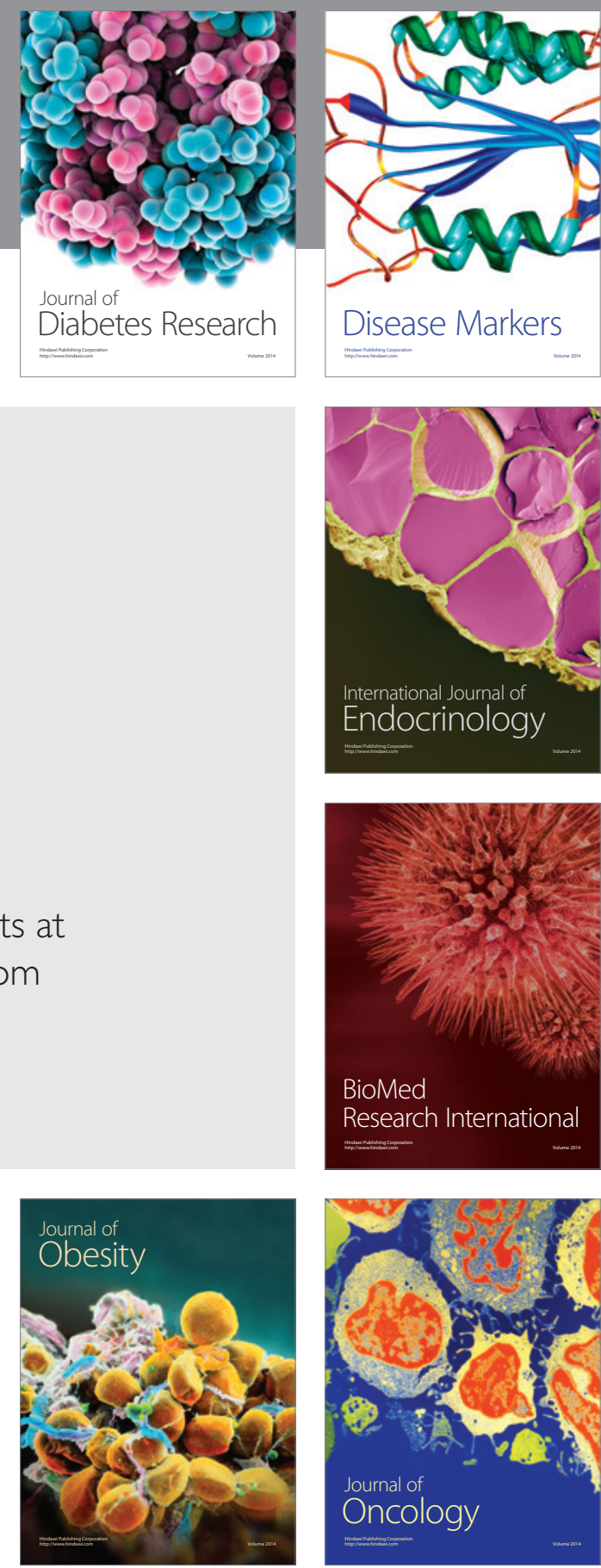

Disease Markers
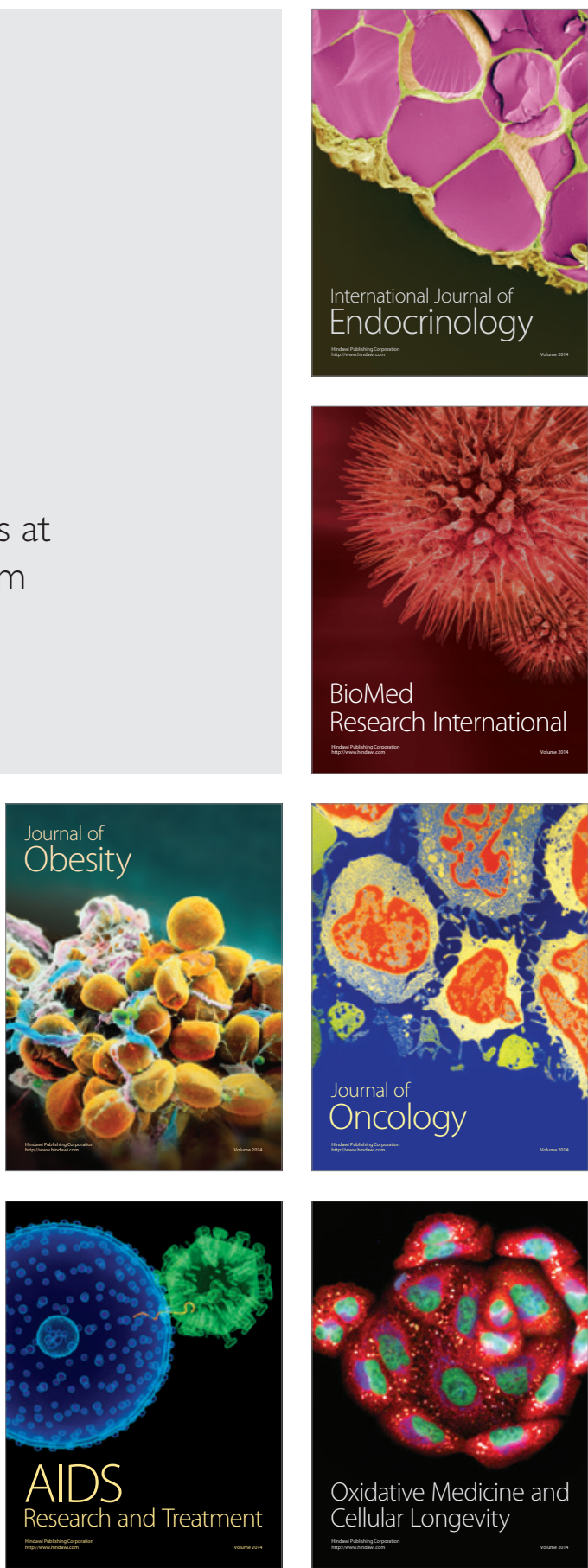